\title{
Training Effects and the Microscopic Magnetic Structure of Exchange
}

\section{Biased Co/CoO Bilayers}

S.G.E. te Velthuis, A. Berger, G.P. Felcher

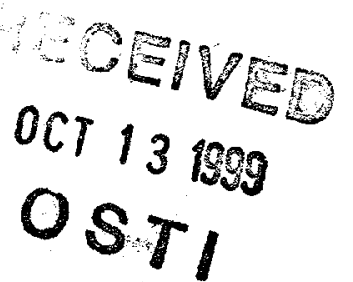

Argonne National Laboratory, Materials Science Division, Bldg. 223, 9700 South Cass Ave., Argonne, IL, 60439, US

B.K. Hill and E. Dan Dahlberg

University of Minnesota, Department of Physics, 116 Church St. SE, Minneapolis, MN, 55455, US

\begin{abstract}
Exchange bias of a partially oxidized thin film of ferromagnetic Co was studied by magnetization measurements and polarized neutron reflectivity (PNR). The magnetization curve shows strong effects of training with cycling of the magnetic field. Reflectivity measurements with the field parallel to the cooling field showed the onset of spin-dependent diffuse scattering -off the specular reflection- after a training cycle. Such scattering, of the Yoneda type, is due to misaligned $\mathrm{Co}$ domains possibly close to the $\mathrm{Co} / \mathrm{CoO}$ interface. Subjecting the field cooled $\mathrm{Co} / \mathrm{CoO}$ pair to a field perpendicular to the cooling field causes a rotation of the magnetization. PNR measurements confirmed earlier susceptibility studies by indicating that the rotation of the magnetization is reversible in fields up to $400 \mathrm{Oe}$. The rotation of the magnetization of $\mathrm{Co}$ is uniform across the film thickness.
\end{abstract}




\section{DISCLAIMER}

This report was prepared as an account of work sponsored by an agency of the United States Government. Neither the United States Government nor any agency thereof, nor any of their employees, make any warranty, express or implied, or assumes any legal liability or responsibility for the accuracy, completeness, or usefulness of any information, apparatus, product, or process disclosed, or represents that its use would not infringe privately owned rights. Reference herein to any specific commercial product, process, or service by trade name, trademark, manufacturer, or otherwise does not necessarily constitute or imply its endorsement, recommendation, or favoring by the United States Government or any agency thereof. The views and opinions of authors expressed herein do not necessarily state or reflect those of the United States Government or any agency thereof. 


\section{DISCLAIMER}

Portions of this document may be illegible in electronic image products. Images are produced from the best available original document. 
Exchange bias was first discovered in 1956 by Meiklejohn and Bean in Co-CoO particle systems [1]. It refers to the occurrence of a unidirectional magnetic anisotropy that manifests itself in shifted hysteresis loops as well as an increase in coercivity for coupled ferromagnet $(\mathrm{F})$-antiferromagnet $(\mathrm{AF})$ systems cooled through the Néel temperature in the presence of a magnetic field [1]. Initially exchange biasing was interpreted as the result of the exchange interaction at AF/F interfaces: the magnitude of the exchange-bias field is given by balancing the gain in Zeeman energy with the energy cost of interfacial exchange when the ferromagnet reverses its magnetization. In the earliest model [2] both $F$ and $A F$ spin structures were assumed to be a rigid sequence of ferromagnetic planes, with an antiparallel sequence for the $\mathrm{AF}$ component; the $\mathrm{AF} / \mathrm{F}$ interface was taken to be atomically flat. Unfortunately such an intuitive picture grossly overestimates the size of the exchange-bias fields. Subsequent models [3-7] attempted to address this difficulty by invoking some roughness at the interface and/or some breakdown in domains of the AF structure.

While traditionally the magnitude of the exchange bias effect has been derived from the shift in the hysteresis curve, it has been argued [7-9] that this method does not evaluate correctly the unidirectional anisotropy. An alternative approach is to obtain the unidirectional anisotropy by means of reversibly rotating the applied field with respect to the exchange bias direction, which is determined by the cooling field $H_{\text {cool }}$. From measurements of this type a value for the exchange anisotropy was derived several times larger than that obtained by the conventional method [8]. In this paper, we confirm with polarized neutron reflectivity (PNR) the reversibility of the rotation of the magnetization of $\mathrm{Co}$ (in a $\mathrm{Co} / \mathrm{CoO}$ thin film) for $H \perp$ $H_{\text {cool }}$ and the rate of rotation away from the bias direction. We find also that such rotation is uniform over the film thickness.

The sample was fabricated by magnetron sputtering of $\mathrm{Co}$ on a Si substrate with a nominal thickness of $120 \AA$. By allowing the sample to oxidize in air a $\mathrm{CoO}$ layer was 
formed. The $\mathrm{Co}$ and $\mathrm{CoO}$ layer thicknesses were determined with X-ray reflectivity, using the $\mathrm{Cu} \mathrm{K} \alpha$ radiation from a rotating anode and were equal to $139 \AA$ and $33 \AA$, respectively. Magnetization measurements were made with a dc magnetometer at $5 \mathrm{~K}$, after cooling from room temperature in a magnetic field $H=5 \mathrm{kOe}$. The field, applied along the cooling field, was cycled five times within $+/-2$ kOe. The training effect is indicated (Fig.1) by the asymmetry of the first cycle and the difference between the first cycle and subsequent cycles. Cooling in different fields (from 1.5 to $30 \mathrm{kOe}$ ) yielded similar results. The symmetric shape of the second cycle permits an estimate of the relevant parameters: exchange bias $H_{E}=145 \pm$ 5 Oe; coercive field $H_{c}=325 \pm 5$ Oe; saturation magnetization $\mathrm{M}=1700 \mathrm{emu} / \mathrm{cm}^{3}$ or close to that of bulk Co. The training effect implies that during the reversal of the magnetization, the ferromagnet does not reverse homogeneously, nor reversibly.

Polarized neutron reflectivity was measured at the POSY I instrument of the Intense Pulsed Neuron Source at Argonne National Laboratory. In order to determine the magnetic profiles of the virgin and trained curves, three measurements at the same field $(H=-65 \mathrm{Oe})$ were made during the first half (I) and the second half (II) of the first hysteresis cycle and during the first half of the second cycle (III), (see Fig.1). The measurements were taken at 4.5 $\mathrm{K}$, after cooling in a field of $5 \mathrm{kOe}$. While the specular reflectivity appears to be fairly similar for all cases, spin-dependent diffuse scattering appears in the case of the trained magnetization. The diffuse scattering is best visible in a contour plot of the intensity as a function of the wavelength $\lambda$ and the angle of incidence $\theta_{i}$ and exit $\theta_{f}$. The contour plot presented in Fig.2 is for neutrons polarized parallel to the applied field for the trained magnetization state (III). For this spin state the reflectivity is higher than for neutrons with opposite polarization (Fig.3) and the diffuse scattering is visible just for this spin state. The diffuse scattering has the characteristics of the "Yoneda scattering" [10-13]. It is distinguished by a ridge of maxima at a critical value of the exit angle, linearly extrapolating 
to the angle of incidence for $\lambda=0$. In Fig.2, a line is drawn for the Yoneda scattering expected if the critical angle is that of the silicon substrate, which corresponds to critical wavenumber $k_{f}=2 \pi \sin \theta_{f} / \lambda=5.05 \times 10^{-3} \AA^{-1}$. The spin dependence of the Yoneda scattering, and its dependence on the magnetic training, indicates that its origin is a magnetic "roughness", if with this name can be called the presence of magnetic domains oriented differently from the main magnetization. The distribution of these domains along the depth of the cobalt layer can in principle be obtain, but requires a careful analysis of the scattered and reflected intensities, presently under way. Much more difficult is to estimate, on the strength of the present data, the lateral extent of such domains.

In order to study the rotation of the magnetization, PNR experiments with polarization analysis of the reflected beam were performed for $H \perp H_{c o o l}$, and with the neutron polarization along the applied field direction. In this case four reflectivities are measured, $R^{--}, R^{-+}, R^{+-}$, and $R^{--}$, where the first sign refers to the polarization state of the incident neutrons, the second to a similar state after reflection. When the magnetization of the sample is perpendicular to the quantization axis of the neutron, the spin-dependent components of the

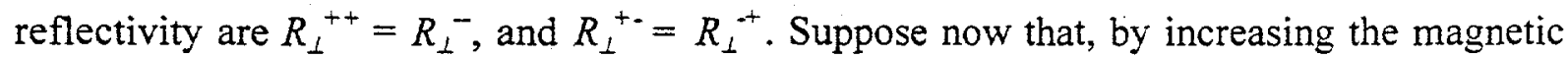
field perpendicular to $H_{c o o l}$, the magnetization is rotated. If the rotation is uniform throughout the sample thickness, then $R^{++} \neq R^{--}$, but

$R^{--}=R^{+\cdot}=R_{\perp}^{-+} \sin ^{2} \alpha$

$\alpha$ is the angle between the applied field and the magnetization. The experimental patterns of $R^{-*}$ at different fields had the same shape over the entire range of neutron momentum transfer $q=$ measured (from 0.01 to $0.08 \AA^{-1}$ ). This indicates the rotation is uniform throughout the Co layer. The scaling factors between runs at different fields can then be interpreted in terms of the angle $\alpha$ between the magnetization and the applied field. The results are presented in Fig.4. 
The rotation of the magnetization is not reversible and displays hysteresis between 0 Oe and 5000 Oe. However, in a field cycle restricted to $0-400 \mathrm{Oe}$, the hysteresis is greatly reduced, indicating that within this field range the rotation of the magnetization is reversible, in agreement with previous ac susceptibility measurements $[8,9]$ on a similar sample. Also similar is the behavior of $\alpha(H)$ found by those two quite different techniques. The unidirectional anisotropy $K_{u d}$ can be estimated with the aid of the expression [9]: $K_{u d}=V M_{s} H \sin (\theta) / A(\theta-\alpha)$,

where $\theta$ is the angle between $H$ and $H_{c o o l}, V$ is the volume, $M_{s}$ is the saturation magnetization of the Co layer and $A$ is the area of the Co-CoO interface. The value obtained from the present data, $K_{u d}=2 \mathrm{erg} / \mathrm{cm}^{2}$ is in good agreement with the quoted value [9] obtained by ac susceptibility.

In conclusion, polarized neutron reflectivity measurements on the rotational hysteresis of a thin film $\mathrm{CoO} / \mathrm{Co}$ confirmed the results previously obtained by ac susceptibility. In weak fields, perpendicular to the cooling field, the rotation of the magnetization is largely reversible. The rotation is found to be uniform throughout the cobalt layer thickness, within the resolution of the measurements which, from the range of $q_{z}$ subtended, is of the order of $20 \AA$. Polarized neutron reflectivity also revealed that training causes a partial breakdown of the alignment of the ferromagnetic domains in intermediate fields. Further studies are needed to establish the position, and possibly the size and correlation of these domains.

Work at Argonne supported by US-DOE, BES-MS contract \#W-31-109-ENG-38, and work at Minnesota supported by NSF/DMR-9809364 


\section{References}

[1] W.H. Meiklejohn and C.P. Bean, Phys. Rev. 105, 904 (1957).

[2] W.H. Meiklejohn, J. Appl. Phys. 33, 1328 (1962).

[3] D. Mauri, H.C. Siegmann, P.S. Bagus, and E. Kay, J. Appl. Phys 62, 3047 (1987).

[4] A.P. Malozemoff, Phys. Rev. B 35, 3679 (1987).

[5] N.C. Koon, Phys. Rev. Lett. 78, 4865 (1997).

[6] T.C. Schulthess and W.H. Bulter, Phys. Rev. Lett. 81, 4516 (1998).

[7] M.D. Stiles and R.D. McMichael, Phys. Rev. B 59, 3722 (1999).

[8] B.H. Miller and E. Dan Dahlberg, Appl. Phys. Lett. 69, 3932 (1996).

[9] Valter Strom, B.J. Jonsson, K.V. Rao, and E. Dan Dahlberg. J. Appl. Phys. 81, 5003 (1997).

[10] Y. Yoneda, Phys. Rev. 131, 2010 (1963).

[11] A. Steyerl, Z. Phys. 254, 169 (1972)

[12] S.K. Sinha, E.B. Sirota, S. Garoff and H.B. Stanley, Phys. Rev. B38, 2297 (1988)

[13] M. Tolan, X-Ray Scattering from Soft-Matter Thin Films, Springer Tracts in Modern Physics \#148, Springer Verlag, Berlin, 1998 


\section{FIGURES}

Figure 1. Magnetizations curve at $T=5 \mathrm{~K}$ after field cooling at $5 \mathrm{kOe}$, measured with $H \|$ $H_{\text {coot. }}$ The virgin (cycle 1) and trained (cycle 2 and 5) magnetization curves are shown. The numbers I, II and III indicate the three magnetization states at which PNR measurement were made.

Figure 2. Linear contour plot of the neutron intensity scattered at grazing incidence measured at $T=4.5 \mathrm{~K}$ for magnetization state III (see Fig.1). The initial neutron polarization is parallel to $H$. The vertical line at $\theta_{i}+\theta_{f}=2 \theta_{i}=2.02^{\circ}$ represents the specular reflectivity. The diagonal line at smaller angle represents the Yoneda scattering from the critical edge of the silicon substrate.

Figure 3. Spin-dependent neutron reflectivity measured (points) and calculated (lines) for incident neutron spin parallel $\left(R^{+}\right)$and antiparallel $\left(R^{-}\right)$to the applied field, at the specular reflection of Fig2. In the insert: approximate profile of the neutron scattering length density (SLD) for the two spin states.

Figure 4 . The spin-flip reflectivity $R^{-+}$normalized on the spin-flip reflectivity at $H=0$ as a function of the applied field $H \perp H_{\text {cool. }}$. The field was increased (decreased) between the data points connected by the solid (dashed) line. The thick solid line represents $\sin ^{2} \alpha$, where $\alpha(\mathrm{H})$ is calculated using Eq. 2 with $\mathrm{K}_{\mathrm{ud}}=2.0 \mathrm{erg} / \mathrm{cm}^{2}$. 


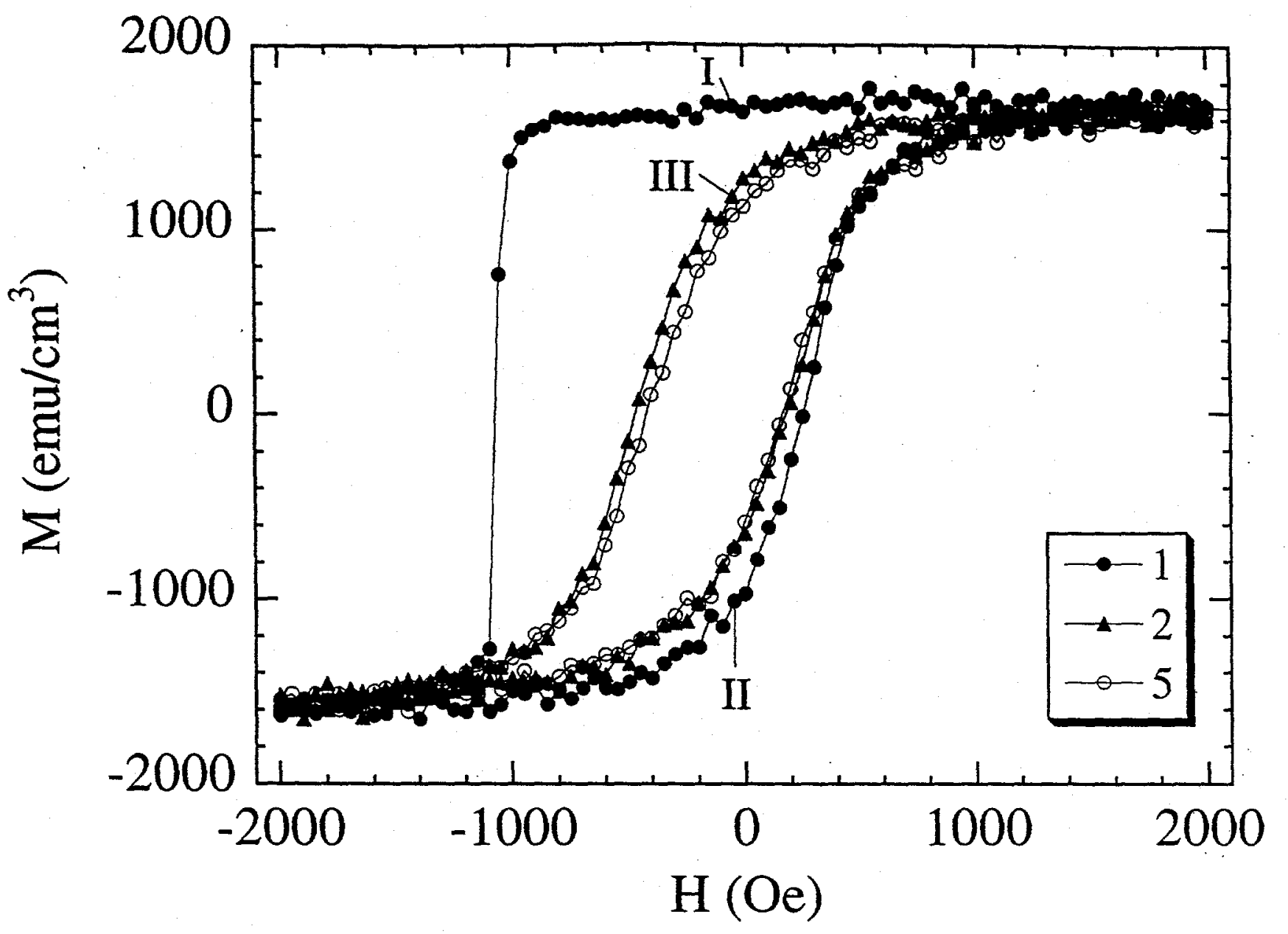

Fig |. te Velthuis JAP 
Fig 2. te velthuis, JAP

Neutron wavelength $(\AA)$

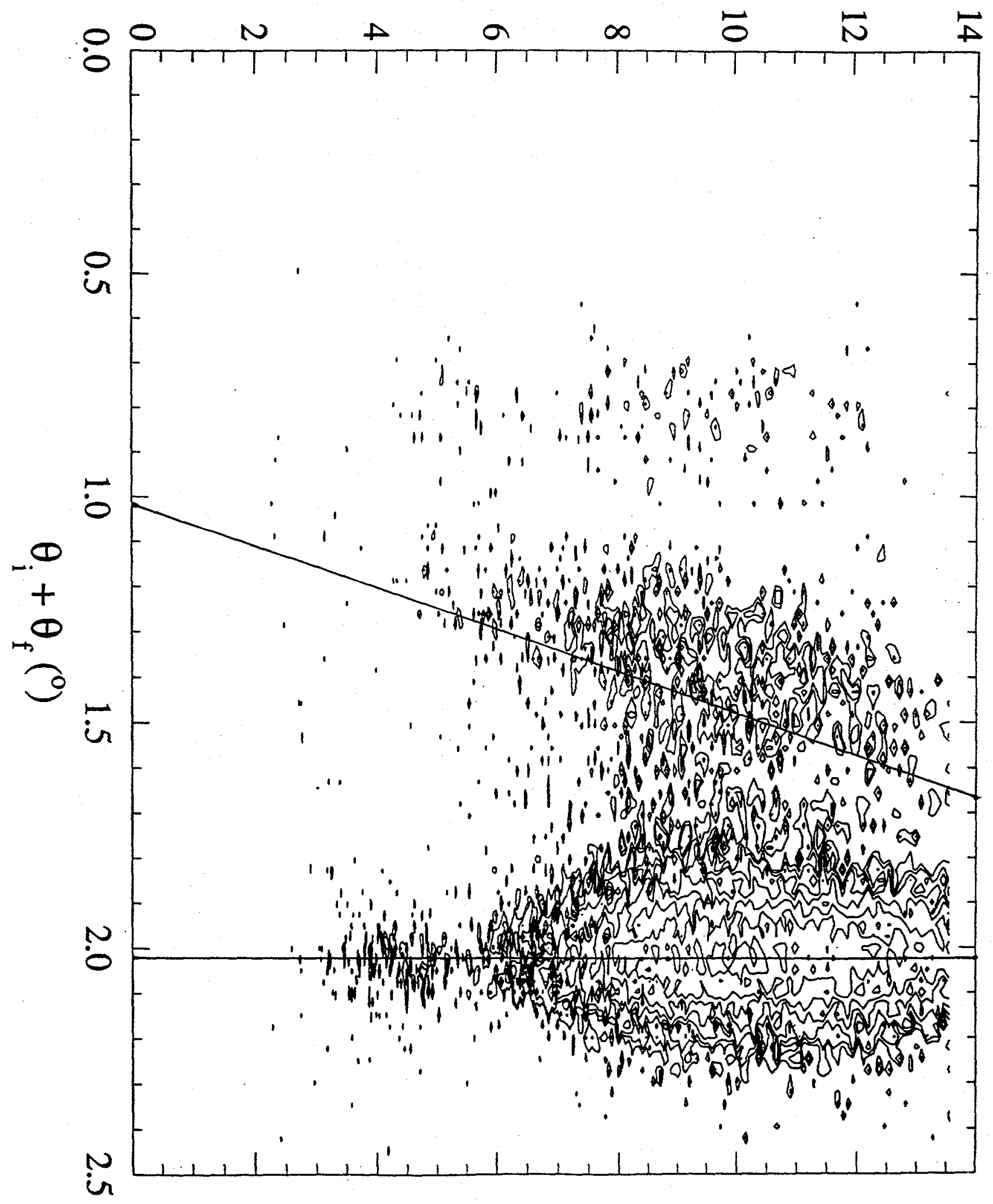




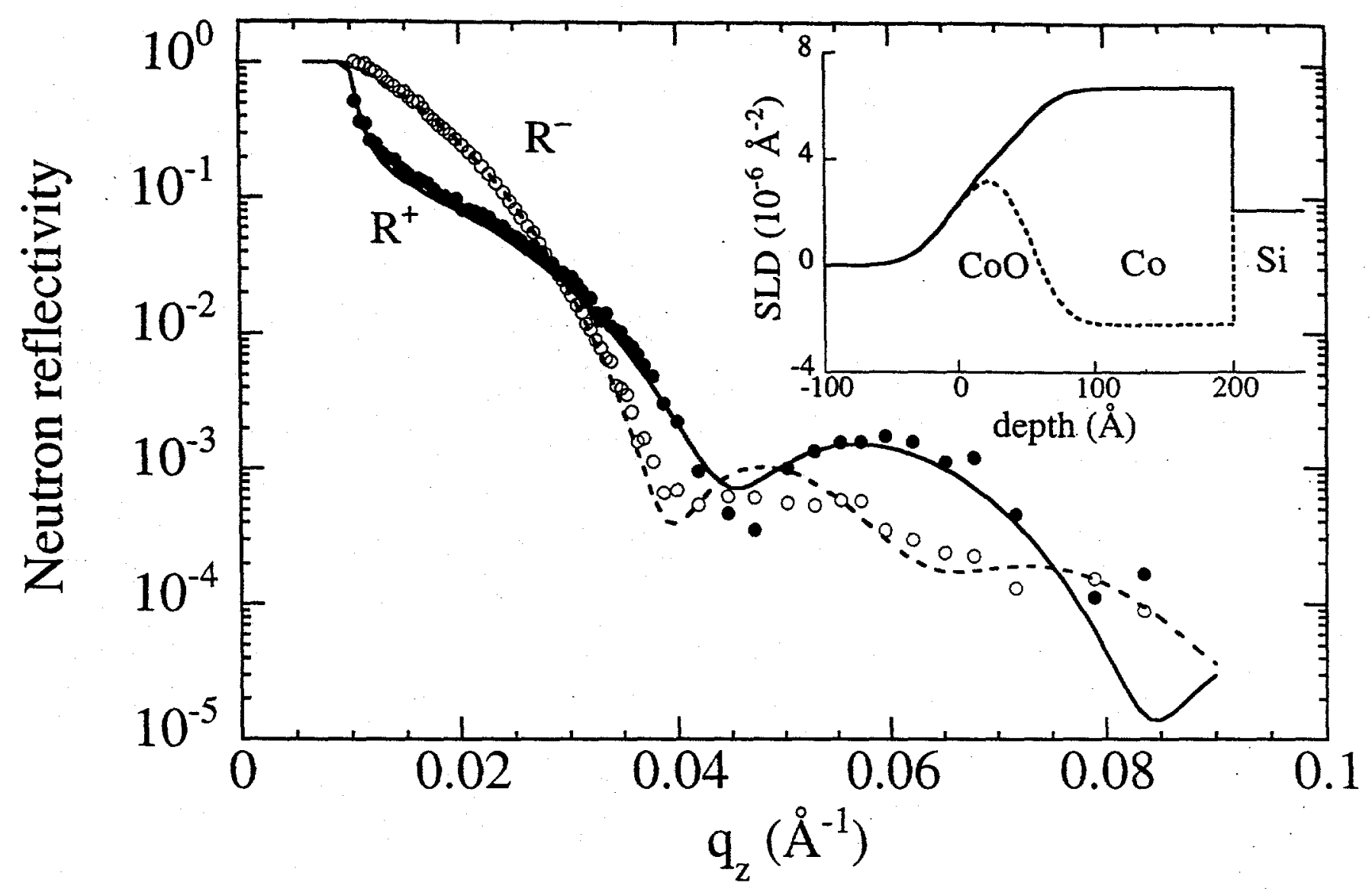




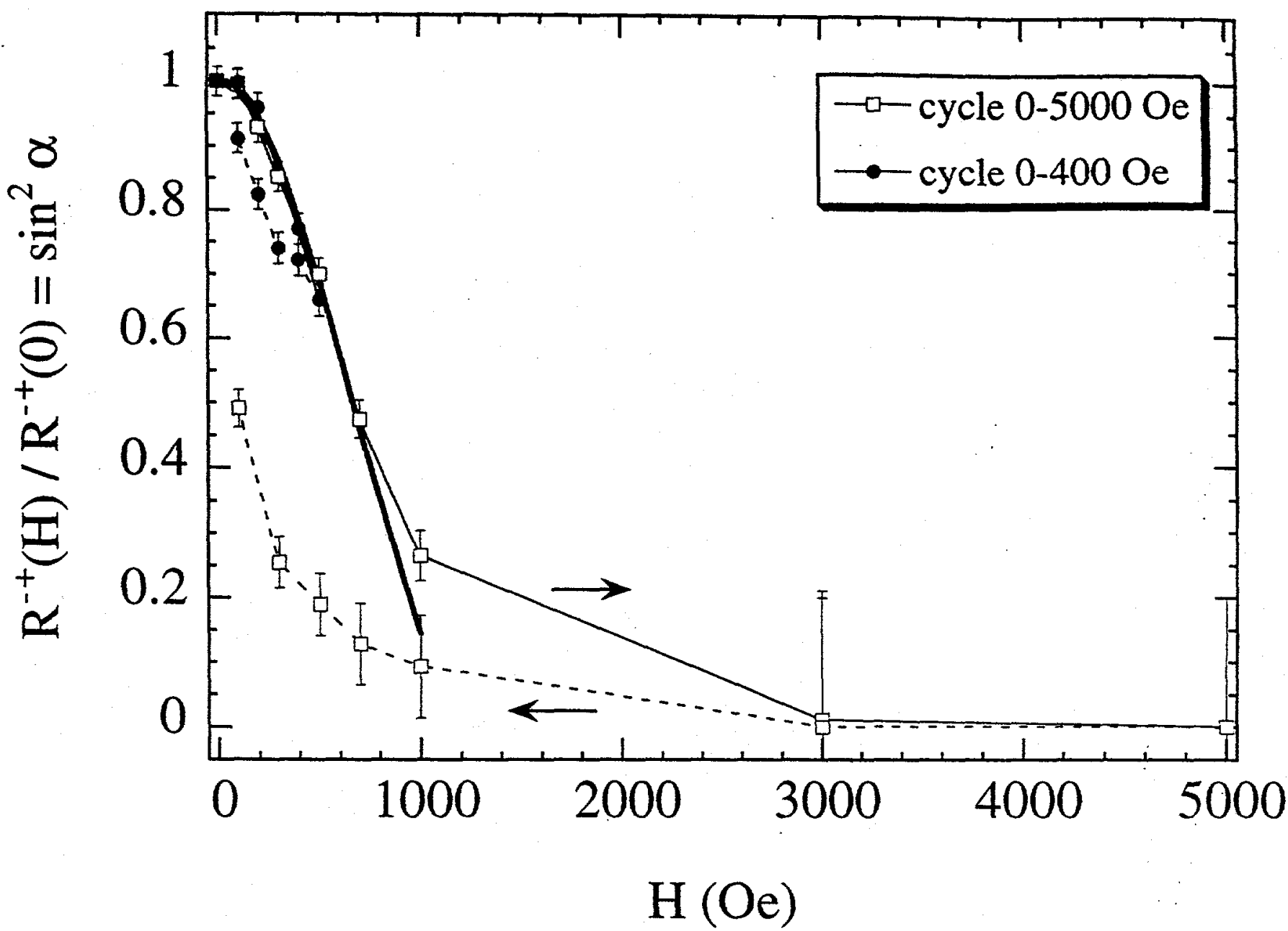

Fig. 4. te Velthuis, JAP 